\title{
Colonic Perforation After Treatment With Nivolumab in Esophageal Cancer: A Case Report
}

\author{
Hye Jung Cho ${ }^{1}$, Woo Ram Kim ${ }^{1}$, Joo-Hang Kim², Duk Hwan Kim³ ${ }^{3}$ Dae Jung Kim ${ }^{4}$, Haeyoun Kang ${ }^{5}$ \\ Departments of ${ }^{1}$ Surgery, ${ }^{2}$ Oncology, ${ }^{4}$ Radiology, ${ }^{5}$ Pathology, and ${ }^{3}$ Digestive Disease Center, CHA Bundang Medical Center, CHA University, \\ Seongnam, Korea
}

With the advent of checkpoint inhibitors, it has opened up opportunities for numerous cancer patients. However, as is the case with every treatment, complications need to be weighed. Gastrointestinal adverse effects, such as diarrhea and colitis are well-known complications for checkpoint inhibitors. In severe cases, colitis-induced colonic perforation may occur with an estimation of $1.0 \%$ to $1.5 \%$ in anti-CTLA- 4 antibodies. However, only a handful of cases of such devastating complications have been reported in anti-PD-1 antibodies such as pembrolizumab and nivolumab. We here report a case of intestinal perforation in a patient treated with nivolumab.

Keywords: Nivolumab; Intestinal perforation; Drug-related side effects and adverse reactions; Immunotherapy

\section{INTRODUCTION}

Cancer immunotherapy opened new horizons for the treatment of various cancers. Immunotherapeutic agents are targeted to prevent cancer immune tolerance, which is acquired in cancer stem cells by suppressing of T-cells via cytotoxic T-lymphocyte-associated antigen 4 (CTLA-4), programmed cell death 1 (PD-1) receptor, and its ligand (PD-L1). With its first approval of anti-CTLA-4 antibodies (ipilimumab) in 2011 by the U.S. Food and Drug Administration for the treatment of advanced melanoma, anti-PD-1 antibodies such as pembrolizumab and nivolumab also received approval in 2014. Ipilimumab (ClinicalTrials.gov registration ID, NCT00094653) showed increased overall survival of 10 months compared to placebo of 6.4 months, and 2-year estimated survival rate was $24 \%$ compared to $14 \%$ [1]. Pembrolizumab and nivolumab also showed promising results in advanced melanoma and nonsmall cell lung cancer (NSCLC).

Received: Mar 26, 2020 - Revised: May 31, 2020 - Accepted: Jun 4, 2020 Correspondence to: Woo Ram Kim, M.D.

Department of Surgery, CHA Bundang Medical Center, CHA University, 59 Yatap-ro, Bundang-gu, Seongnam 13496, Korea

Tel: +82-31-780-1824, Fax: +82-31-780-5259

E-mail: christtome81@chamc.co.kr

ORCID: https://orcid.org/0000-0003-1651-3778

(C) 2021 The Korean Society of Coloproctology

This is an open-access article distributed under the terms of the Creative Commons Attribution NonCommercial License (https://creativecommons.org/licenses/by-nc/4.0) which permits unrestricted noncommercial use, distribution, and reproduction in any medium, provided the original work is properly cited.
Although PD-L1 is expressed only in 30\% to $50 \%$ of gastrointestinal cancers [2], clinical trials are underway in advanced gastric cancer $[3,4]$. As the scope of its use increases, unforeseen side effects known as immune-related adverse events (irAEs) emerge in dermatologic, gastrointestinal, and endocrine systems [5]. Although most events subside with appropriate use of immunosuppressive treatment, severe cases may lead to intestinal perforation. We here report what we know as the third case of intestinal perforation shortly after initiation of nivolumab, an anti-PD-1 monoclonal antibody.

\section{CASE REPORT}

A 58-year-old male was diagnosed with esophageal cancer in April 2016 and was treated with esophagogastrostomy and adjuvant chemotherapy (capecitabine) for 2 months. Other surgical history includes laparoscopic cholecystectomy and 3 diagnostic laparoscopies for peritonitis, adhesive ileus, and internal hernia. The patient underwent follow-ups every 6 months and showed no progression for 2 years. In December 2018, the patient visited the oncology clinic a month earlier than his scheduled visit complaining of hoarseness. Further evaluation including neck computed tomography (CT) and positron emission tomography-CT showed recurred esophageal cancer. The patient was started on radical concurrent chemoradiation therapy of $60 \mathrm{~Gy} / 30 \mathrm{Fx}$ and weekly cisplatin. Evaluation showed partial response, and docetaxel (dose reduction, 25\%) for 3 months showed stable disease. 


\section{$\begin{array}{rr}\text { Annals of } & \text { Colonic Perforation } \\ \text { Coloproctology } & \text { Hye Jung Cho, et al. }\end{array}$}

After further discussion, in August 2018, the patient was started on nivolumab (3 mg/kg every 2 weeks). After his second dose of nivolumab treatment, the patient complained of diarrhea and abdominal pain. Upon his admission for his third dose, nivolumab was held due to possible colitis. Conservative treatment including steroid therapy (prednisolone $1 \mathrm{mg} / \mathrm{kg} /$ day per oral, later increased to intravenous [IV] methylprednisolone $3 \mathrm{mg} / \mathrm{kg} /$ day) was initiated, but symptoms continued. Every possible differential diagnosis was required, including cytomegalovirus (CMV). Generally, immunocompromised patients undergoing chemotherapy are susceptible to CMV colitis. Infection workup including stool culture, Clostridioides difficile antigen, and viral studies including CMV were performed, and sigmoidoscopy revealed various-sized multiple discrete ulcerations with normal intervening mucosa from sigmoid colon to rectum (Fig. 1). Biopsy from the sigmoid showed chronic, nonspecific inflammation with ulceration showing moderate lamina proprial lymphoplasmacytic infiltration (Fig. 2A). Evaluating the shape and character of the ulcers, our endoscopist concluded the possibility of CMV colitis and performed immunohistochemical stain for $\mathrm{CMV}$, which was negative. However, $\mathrm{CMV}$ polymerase chain reaction (PCR) performed via blood sample was positive with negative CMV immunoglobulin (Ig) $\mathrm{M}$ and elevated CMV IgG of $32 \mathrm{AU} / \mathrm{mL}$ (Fig. 2B). We further evaluated for acute CMV infection via quantitative CMV PCR of the blood, and the results showed less than detection limit.

Prior to the results of the CMV PCR and the biopsy result, IV ganciclovir was added to the treatment with the impression of $\mathrm{CMV}$ colitis. The result of the immunohistochemistry and the bi-
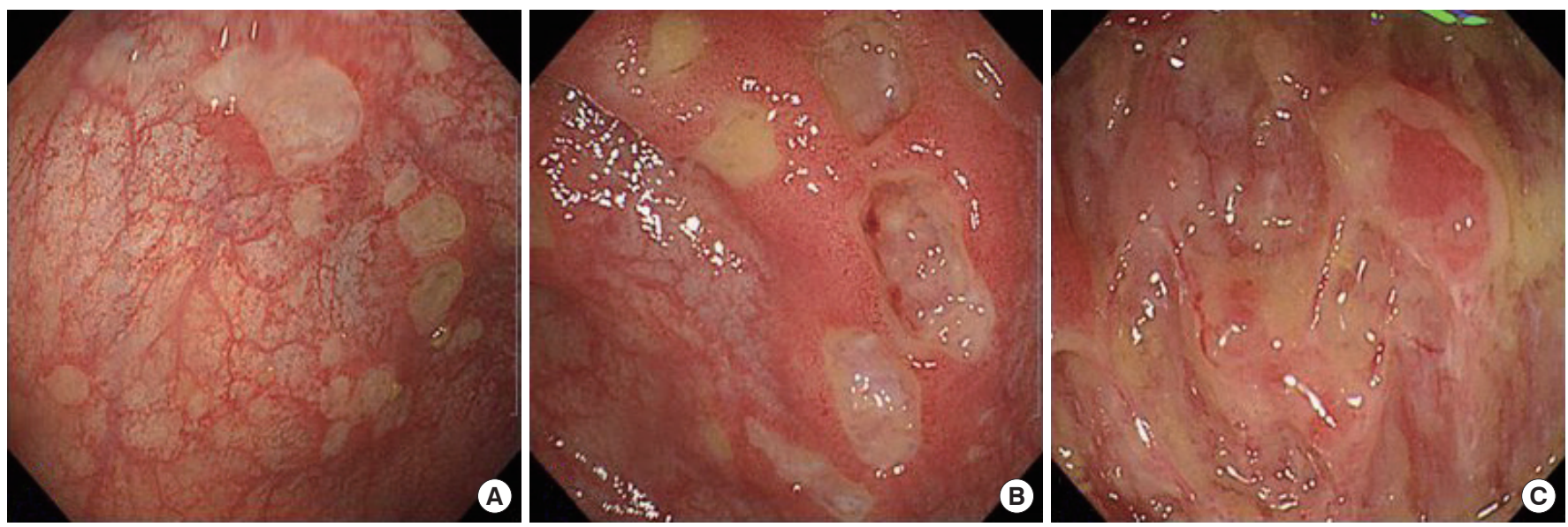

Fig. 1. Sigmoidoscopic findings. (A) Multiple shallow discrete ulcerations with variable size in the rectum. (B) Deep oval punched-out ulcerations in the sigmoid colon. (C) Large ulceration with irregular ulcer base in the sigmoid colon.
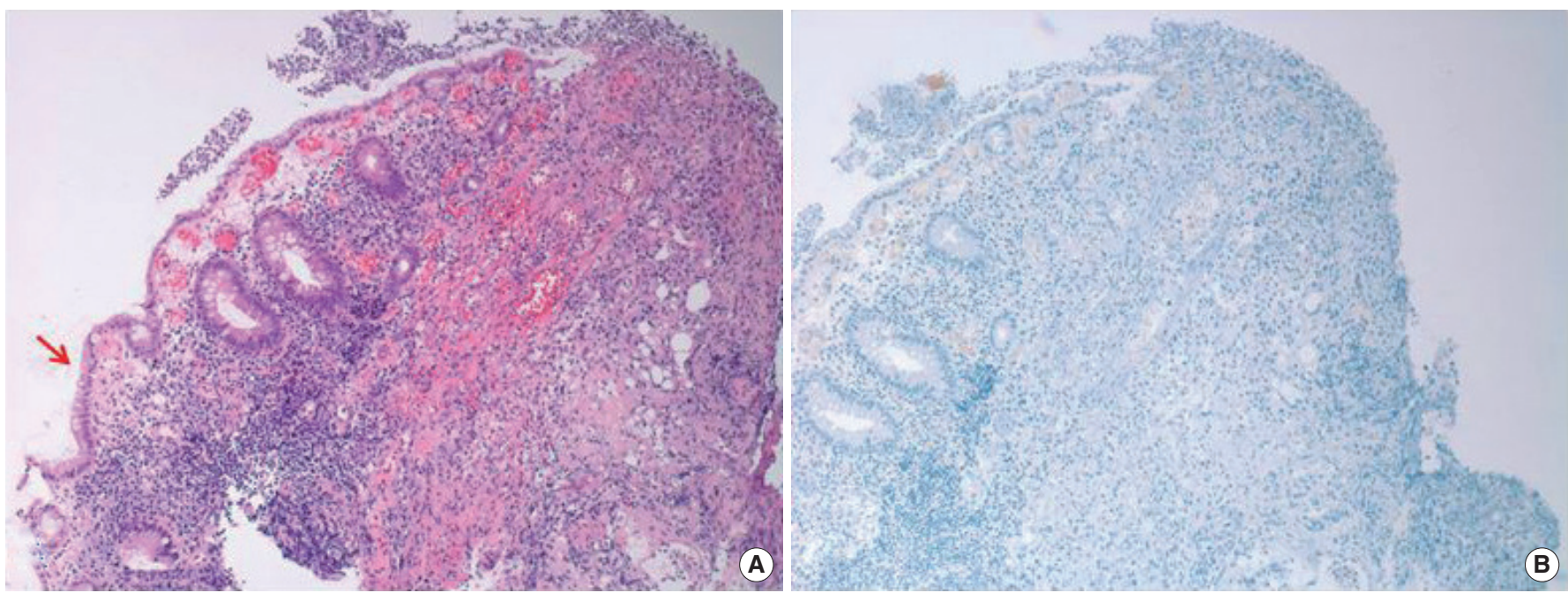

Fig. 2. Pathological findings. (A) Sigmoid colon biopsy shows chronic inflammation with ulceration showing moderate lamina proprial lymphoplasmacytic infiltration (arrow) (H\&E, $\times 100)$. (B) The immunohistochemical stain for cytomegalovirus was negative $(\times 100)$. 
Volume 37, Supplement 1, 2021

opsy result were reported 1 week after the biopsy. These tests usually take 7 days to final report. During this period, the patient's symptoms were aggravated despite treatment with steroids and antibiotics. The oncology department made clinical decision to empirically treat CMV colitis. After being transferred to general surgery department, the patient underwent emergency operation and the result of the immunohistochemistry staining was negative. We then stopped the treatment of gancyclovir. Unresolved abdominal pain warranted abdominal CT, and multifocal pneumoperitoneum with possible bowel perforation was diagnosed (Fig. 3B, C). General surgery department was consulted for emergency operation.

The patient had stable vital signs with no fever, but the use of steroids may have masked the symptoms. Physical examination showed tenderness on right lower quadrant with mild muscle guarding. Laboratory results revealed following results; white blood cells of $12,080 / \mu \mathrm{L}$, hemoglobin of $12.0 \mathrm{~g} / \mathrm{dL}$, platelet of $652,000 / \mu \mathrm{L}$, albumin of $1.9 \mathrm{~g} / \mathrm{dL}$, and C-reactive protein of $21.76 \mathrm{mg} / \mathrm{dL}$ (normal range, $<0.3 \mathrm{mg} / \mathrm{dL}$ ). Emergency surgery was planned for diagnostic laparoscopy.

Intraoperatively, severe adhesion and yellowish exudate of the distal ileum and the cecum were observed in the right lower quadrant (Fig. 4A). Careful dissection of the adhesion revealed a 5-mm defect on the cecum wall (Fig. 4C). Primary closure was carried out laparoscopically (Fig. 4D). In addition, multiple ulcerations were visualized along the sigmoid colon, which we deemed as possible future perforation sites (Fig. 4B). After much discussion, we decided it would be safe and necessary to maturate a loop ileostomy until colitis was resolved. The patient recovered well in our surgical unit and began oral intake on postoperative day (POD) 1.

On POD 6, the patient was transferred to the oncology unit for further treatment. Prior to the surgery, the patient complained of epigastric pain, which was treated with proton-pump inhibitors. However, his hoarseness continued even after the surgery, which warranted further evaluation. On POD 8, chest CT was performed, which revealed a possible abscess formation in the previous esophagogastrotomy site. Magnetic resonance imaging revealed a minor perforated esophagogastrostomy site at $\mathrm{T} 1$ level with continuous
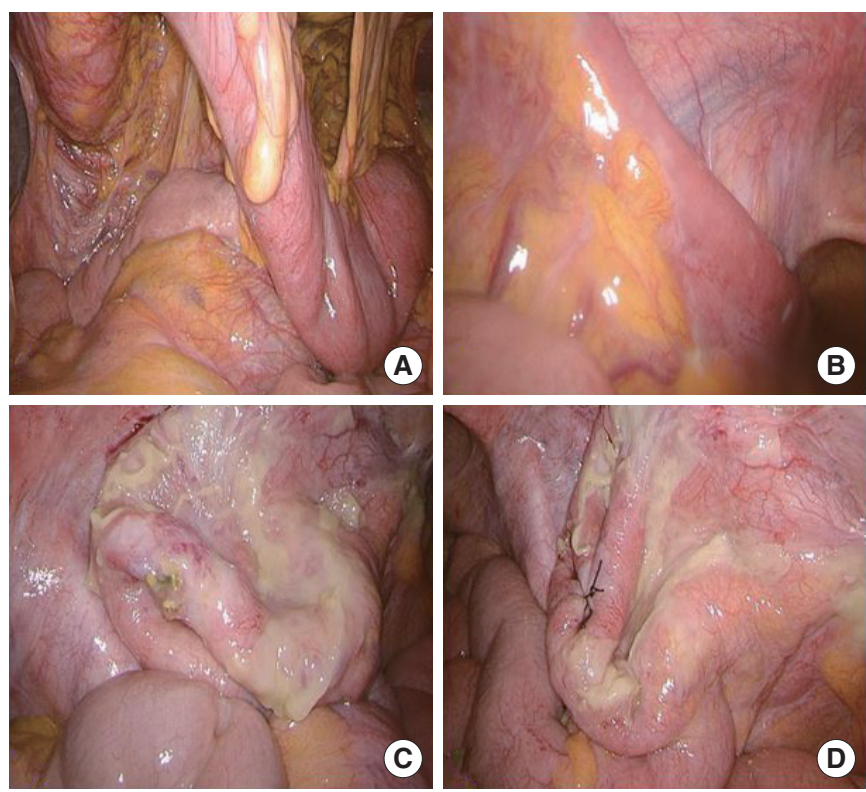

Fig. 4. Intraoperative findings. (A) Abdominal wall adhesions of perforated cecum. (B) Multiple ulcerations in sigmoid colon. (C) Perforation at the cecum with severe inflammation. (D) Laparoscopic primary repair of the cecal perforation.
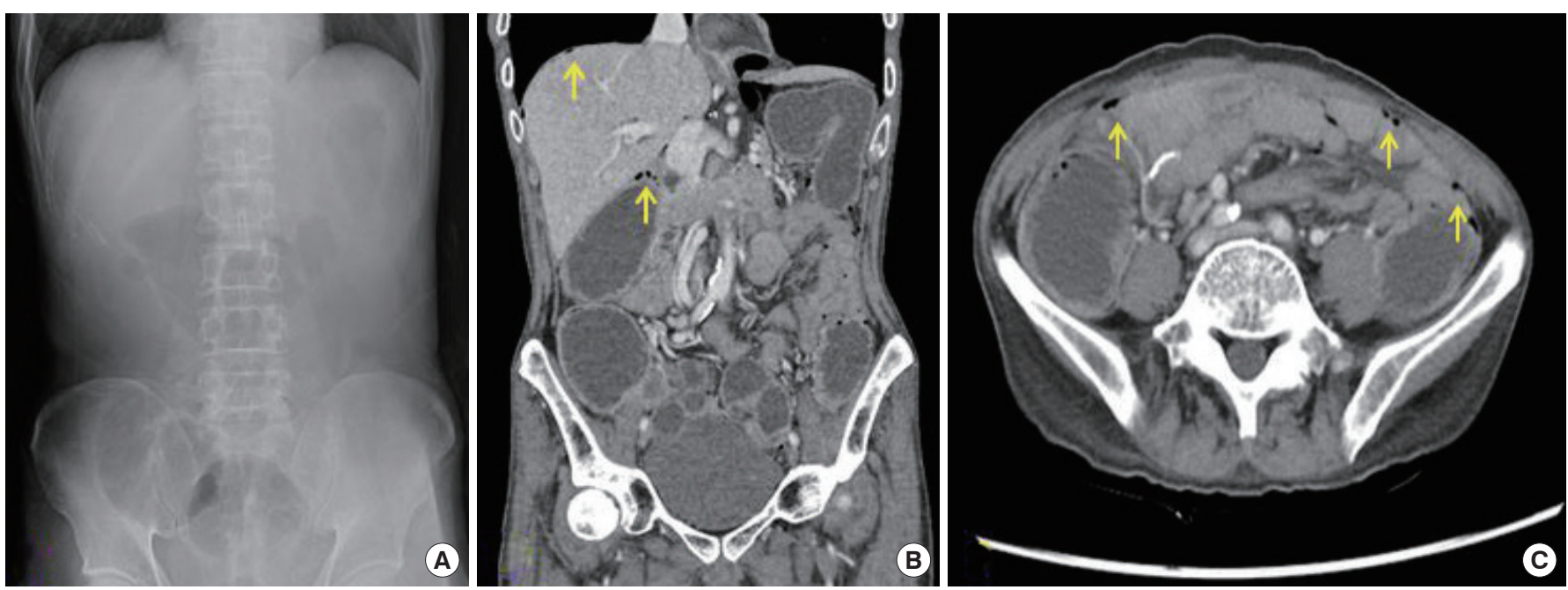

Fig. 3. Imaging studies. (A) Abdomen X-ray. (B, C) Abdominal computed tomography of coronal (B) and axial (C) view. Pneumoperitoneum (arrows) is shown. 
air pocket at left lateral side of the trachea. The patient was transferred to the hospital at which he underwent his initial esophagogastrostomy. His esophagogastroduodenoscopy revealed perforation near upper esophageal sphincter, which excluded the patient from stent insertion. He underwent conservative management with total parental nutrition and antibiotics, and went into hospice care. The patient expired in March of 2020 and was not able to ileostomy repair.

This study was approved by the Institutional Review Board (IRB) of CHA Bundang Medical Center and informed consent was waived by the IRB (No. 2020-01-014).

\section{DISCUSSION}

Immeasurable amount of advancements in the treatment of cancer have been developed with the latest trend focusing on the immune checkpoint inhibitors. Generally approved for advanced melanoma, NSCLC, and renal cell carcinoma, doors are opening to other types of cancers.

With benefits comes cost known as irAEs. Symptoms range from rash, diarrhea, hepatitis, hypophysitis, adrenal crisis to inflammatory pneumonia, renal insufficiency, pancreatitis, encephalopathy, and neutropenia [5]. Gastrointestinal side effects, such as diarrhea and colitis are quite common. Severe diarrhea (grade 3 or 4) occurs more commonly in anti-CTLA-4 antibodies than in anti-PD-1 or anti-PD-L1 antibodies; $10 \%$ compared to $1 \%-2 \%$ [5]. With the use of the combination of anti-CTLA-4 and anti-PD-L1 antibodies, the rate increases dramatically. Onset of gastrointestinal symptoms usually presents after 6 weeks of treatment but can arise at any time after initiation of treatment $[5,6]$. Typically, patients with mild diarrhea improve with hydration and corticosteroids. In severe cases, IV corticosteroids (up to $2 \mathrm{mg} / \mathrm{kg}$ methylprednisolone twice a day) or if resistant, infliximab (5 mg/kg, once every 2 weeks) may be utilized. However, in cases of diarrhea and abdominal pain that persists for more than 1 week, rectosigmoidoscopy should be performed along with screening for $C$. difficile and CMV [7]. Because the endoscopic findings are nonspecific, ranging from erythema, edema, and erosion to ulceration, bleeding, graininess, and loss of vascularization, biopsies should be routine [6-8]. Histologically, immune checkpoint inhibitor-induced colitis usually presents with acute focal or patchy areas of inflammation with infiltrating eosinophil and neutrophils and abscesses within the crypts [6].

With up to $50 \%$ of patients on anti-CTLA- 4 antibodies exhibiting signs of diarrhea, ipilimumab-induced colitis is a well-known side effect that should be explained to the patient prior to initiating treatment. In severe cases, colitis-induced colonic perforation is estimated to occur in $1.0 \%$ to $1.5 \%$ of patients treated with ipilimumab [8]. However, very little is known on gastrointestinal irAEs related to anti-PD-1 antibodies; nivolumab and pembrolizumab. Endoscopic findings are similar to that of anti-CTLA-4 antibodies-induced colitis, but histological findings include lamina proprial expansion, villous blunting, intraepithelial neutrophils/lym- phocytes, and increased crypt/gland apoptosis [6]. A study conducted by Chen et al. [9] reviewing histopathology of 8 cases of PD-1 inhibitor-associated colitis. Five patients showed acute colitis with crypt abscesses and epithelial cell apoptosis while 3 cases showed intraepithelial lymphocytic colitis. Our patient's histology showed chronic nonspecific inflammation with ulceration and moderate lamina proprial lymphoplasmacytic infiltration, and the immunohistochemical staining was negative for CMV, quite similar to the later histological features mentioned above.

The most common irAEs of nivolumab are fatigue, pruritus, and nausea with no treatment-related death and rarely severe complications of grade 3 or higher [1]. Malipatil et al. [10] reported the first case of nivolumab-induced gastric perforation leading to discontinuation of treatment. Yasuda et al. [11] reported the first intestinal perforation of the ileum after 3 cycles of nivolumab treatment for malignant melanoma. Other cases of intestinal perforation cases have been reported, but these cases were induced by anti-CTLA- 4 antibodies. There were 2 other cases where combination of anti-CTLA-4 and anti-PD-L1 antibodies were used [12, 13]. To our knowledge, this is the third case of anti-PD-1 monotherapy induced intestinal perforation.

Although immunohistochemical staining of CMV during the colonoscopy was negative, CMV PCR of blood was positive with elevated CMV IgG level. CMV reactivation after immunotherapy has been reported by Lankes et al. [14]; however, with CMV DNA levels below detection limit and CMV IgM being negative, reactivation is unlikely [14]. With moderate lamina proprial lymphoplasmacytic infiltration shown in histology and the initiation of gastrointestinal symptoms shortly after nivolumab treatment, we cautiously deduce nivolumab-induced colonic perforation. Although further accumulation of cases and clinical trials are required, nivolumab-induced colitis leading to gastrointestinal perforation is not mundane but can arise. Patients not only on anti-CTLA-4 antibodies but also in anti-PD-1 or anti-PD-L1 antibodies should be advised of the side effects and be monitored for early signs.

\section{CONFLICT OF INTEREST}

No potential conflict of interest relevant to this article was reported.

\section{REFERENCES}

1. La-Beck NM, Jean GW, Huynh C, Alzghari SK, Lowe DB. Immune checkpoint inhibitors: new insights and current place in cancer therapy. Pharmacotherapy 2015;35:963-76.

2. de Guillebon E, Roussille P, Frouin E, Tougeron D. Anti program death-1/anti program death-ligand 1 in digestive cancers. World J Gastrointest Oncol 2015;7:95-101.

3. Bang YJ, Kang YK, Catenacci DV, Muro K, Fuchs CS, Geva R, et al. Pembrolizumab alone or in combination with chemotherapy as first-line therapy for patients with advanced gastric or gastro- 
esophageal junction adenocarcinoma: results from the phase II nonrandomized KEYNOTE-059 study. Gastric Cancer 2019;22: 828-37.

4. Shitara K, Özgüroğlu M, Bang YJ, Di Bartolomeo M, Mandalà M, Ryu $\mathrm{MH}$, et al. Pembrolizumab versus paclitaxel for previously treated, advanced gastric or gastro-oesophageal junction cancer (KEYNOTE-061): a randomised, open-label, controlled, phase 3 trial. Lancet 2018;392:123-33.

5. Postow MA. Managing immune checkpoint-blocking antibody side effects. Am Soc Clin Oncol Educ Book 2015:76-83.

6. Haanen JB, Carbonnel F, Robert C, Kerr KM, Peters S, Larkin J, et al. Management of toxicities from immunotherapy: ESMO Clinical Practice Guidelines for diagnosis, treatment and follow-up. Ann Oncol 2018;29(Suppl 4):iv264-6.

7. Pernot S, Ramtohul T, Taieb J. Checkpoint inhibitors and gastrointestinal immune-related adverse events. Curr Opin Oncol 2016; 28:264-8.

8. Beck TN, Kudinov AE, Dulaimi E, Boumber Y. Case report: reinitiating pembrolizumab treatment after small bowel perforation.
BMC Cancer 2019;19:379.

9. Chen JH, Pezhouh MK, Lauwers GY, Masia R. Histopathologic features of colitis due to immunotherapy with anti-PD-1 antibodies. Am J Surg Pathol 2017;41:643-54.

10. Malipatil B, Palleti A, Verma NS, Katti SV. Gastric perforation due to nivolumab related tumor flare. Indian J Cancer 2019;56:374-5.

11. Yasuda K, Tanaka T, Ishihara S, Otani K, Nishikawa T, Kiyomatsu $\mathrm{T}$, et al. Intestinal perforation after nivolumab immunotherapy for a malignant melanoma: a case report. Surg Case Rep 2017;3:94.

12. Celli R, Kluger HM, Zhang X. Anti-PD-1 therapy-associated perforating colitis. Case Rep Gastrointest Med 2018;2018:3406437.

13. Delasos L, Desai A, Lopetegui Lia N, Kethireddy N, Ray C. A case of immunotherapy-induced colitis complicated by perforation and treated with infliximab postoperatively. Case Rep Oncol Med 2019; 2019:9069354.

14. Lankes K, Hundorfean G, Harrer T, Pommer AJ, Agaimy A, Angelovska I, et al. Anti-TNF-refractory colitis after checkpoint inhibitor therapy: possible role of CMV-mediated immunopathogenesis. Oncoimmunology 2016;5:e1128611. 